\title{
Fatores relacionados à dependência do smartphone em adolescentes de uma região do Nordeste brasileiro
}

\author{
Factors related to smartphone addiction \\ in adolescents from a region in Northeastern Brazil
}

Paula Pessoa de Brito Nunes (https://orcid.org/0000-0002-7462-0494) 1,2 Ana Paula Vasconcellos Abdon (https://orcid.org/0000-0002-1597-1817) ${ }^{1}$ Claudiana Batista de Brito (https://orcid.org/0000-0002-1827-8276) ${ }^{2}$

Francisco Valter Miranda Silva (https://orcid.org/0000-0002-7122-2466) 1 Ionara Conceição Araújo Santos (https://orcid.org/0000-0002-9484-2078) ${ }^{2}$ Daniele de Queiroz Martins (https://orcid.org/0000-0001-5732-4581) ${ }^{2}$ Phelipe Maia Fonseca Meira (https://orcid.org/0000-0002-1153-2665) ${ }^{2}$ Mirna Albuquerque Frota (https://orcid.org/0000-0003-3004-2554) ${ }^{1}$

${ }^{1}$ Programa de PósGraduação em Saúde Coletiva, Universidade de Fortaleza. Av. Washington Soares 1321 Bloco S Sala 1 , Edson Queiroz. 60811905 Fortaleza CE Brasil. paulanunes2912@ gmail.com

${ }^{2}$ Centro Universitário UniAteneu. Fortaleza CE Brasil.

\begin{abstract}
Adverse effects on the physical and mental health of adolescents associated with excessive smartphone use have been reported. This paper aims to assess adolescent smartphone addiction and related factors in a region in Northeastern Brazil. This cross-sectional study was carried out in six State-run Professional Education Schools in Fortaleza, Ceará, Brazil, from September to October 2019 with 286 adolescents aged 15-19 years who completed five data collection instruments. Bivariate and multivariate analyses were performed to assess factors related to the outcome using SPSS version 23.0. Smartphone addiction prevalence rate was $70,3 \%$ and was associated with being underage $(O R=0,583 ; p=0,001)$, fewer sleep hours $(O R=0,715 ; p=0,020)$, longer use on weekends (OR=1,115; $p=0,015)$, cervical pain $(O R=2,206 ; p=0,020)$, and suspected common mental disorder $(O R=1,272 ; p=0,000)$. A high smartphone addiction level was observed among adolescents in the sample and was related to multiple factors. Attention should be drawn to the importance of educational campaigns to guide adolescents, parents, educators, and health professionals about the risks of excessive smartphone use to the health of adolescents.
\end{abstract}

Key words Addiction medicine, Smartphone, Risk factors, Adolescents
Resumo Há relatos de efeitos adversos na saúde física e mental dos adolescentes associados ao uso excessivo do smartphone. O objetivo deste artigo é avaliar a dependência do smartphone e os fatores relacionados em adolescentes de uma região do Nordeste brasileiro. Trata-se de estudo transversal realizado em seis Escolas Estaduais de Educação Profissional (EEEPs), na cidade de Fortaleza, Ceará, Brasil, desenvolvido entre setembro e outubro de 2019. Participaram 286 adolescentes, entre 15 e 19 anos, que responderam cinco instrumentos de coleta. Análises bivariada e multivariada foram utilizadas para avaliar os fatores relacionados ao desfecho, pelo SPSS versão 23.0. A dependência do smartphone apresentou prevalência de $70,3 \%$, e mostrava associação com menor idade (OR=0,583; $p=0,001)$, menos horas de sono $(\mathrm{OR}=0,715 ; \mathrm{p}=0,020)$, mais tempo de uso no final de semana (OR=1,115; $p=0,015)$, queixa de dor cervical $(O R=2,206 ; p=0,020)$ e suspeita de transtorno mental comum $(O R=1,272 ; p=0,000)$. Evidenciou-se elevada dependência do smartphone nos adolescentes da amostra, relacionada a múltiplos fatores. Alerta-se para a importância de campanhas educativas que orientem os adolescentes, pais, educadores e profissionais de saúde para os riscos do uso excessivo de smartphones à saúde dos adolescentes.

Palavras-chave Medicina do vício, Smartphone, Fatores de risco, Adolescentes 


\section{Introdução}

Nas últimas décadas, vive-se uma transição social que vem modificando a sociedade em seu jeito de pensar, comunicar, relacionar e trabalhar. Essas mudanças foram geradas pela transformação digital. A tecnologia possibilita diferentes pessoas se comunicarem ao mesmo tempo, conectadas em uma única rede, porém distantes fisicamente. Dentre os dispositivos tecnológicos, o smartphone é um dos mais utilizados para acessar a internet ${ }^{1}$.

Em todo o mundo, cresce de forma exponencial o número de usuários de smartphones independentemente da idade ${ }^{2}$. Dados apontam aumento de $54 \%$ em seu uso como principal forma de acesso à internet pela população, segundo levantamento em 21 países emergentes ${ }^{3}$. Neste cenário, o Brasil ocupa o segundo lugar no ranking mundial em relação ao tempo diário conectado à internet, de 9 horas por $\mathrm{dia}^{4}$. Outro inquérito direcionado para crianças e adolescentes informa que $86 \%$ acessam a internet por este dispositivo ${ }^{5}$.

Tal crescimento está associado a inúmeros recursos como comunicação e entretenimento, que são compactados no mesmo dispositivo ${ }^{6}$. A facilidade de acesso apresenta vantagens como aumento da produtividade, velocidade da propagação de informações e maior prazer no uso das redes sociais. Estudos mostram que a população utiliza o smartphone de forma excessiva, destacando os adolescentes como grupo de risco quanto ao uso prolongado ${ }^{7}$.

Este dispositivo oferece conveniências aos seus usuários, no entanto seu uso prolongado causa problemas que comprometem as condições de saúde ${ }^{8}$. A literatura descreve a dependência do smartphone como distúrbio comportamental caracterizado pela impulsividade e pelo uso incontrolável como visualização constante de notificações, jogos e aplicativos, sendo comparado a outras dependências comportamentais. Apesar de não existir uma classificação específica dentro do Manual de Diagnóstico e Estatística de Transtornos Mentais (DSM-5), este comportamento gera consequências negativas nos aspectos biopsicossociais dos adolescentes 9 .

Os adolescentes são fortemente vinculados aos seus smartphones, e se dizem incapazes de viver sem seus dispositivos. Considerados como nativos digitais, muitas vezes os utilizam de maneira dependente seguindo a moda no uso de aplicativos e em busca de relacionamentos e apoio emocional. Tais jovens, comumente, expressam seus pensamentos em um ambiente de recreação virtual, à procura de reações e feedbacks instantâneos ${ }^{10}$.

Frente ao cenário apresentado, destaca-se a necessidade de fomentar dados referentes ao uso excessivo do smartphone e suas consequências para direcionar ações e preencher lacunas, conforme previamente alerta a Organização Mundial da Saúde $(\mathrm{OMS})^{11}$. Diante disso, este estudo teve como objetivo avaliar a dependência do smartphone e os fatores relacionados em adolescentes de uma região do Nordeste brasileiro.

\section{Métodos}

Trata-se de um estudo quantitativo, do tipo transversal e analítico, advindo de um projeto de pesquisa intitulado "Estudo das alterações posturais e álgicas na região cervical associada ao uso de smartphone em adolescentes", desenvolvido em Escolas Estaduais de Educação Profissional (EEEPs) de Ensino Médio da cidade de Fortaleza, Ceará, Brasil. O recrutamento e coleta de dados foram realizados nos meses de agosto e setembro de 2019.

A cidade de Fortaleza, capital do estado do Ceará, é a quinta mais populosa do Brasil com uma população de 2.643 .247 pessoas. Seu Índice de Desenvolvimento Humano Municipal (IDHM) é de 0,754 e de Educação é de 0,672, assumindo a $17^{\text {a }}$ colocação do ranking do IDH das metrópoles do país ${ }^{12}$. Desde 1997, está dividida administrativamente em sete Secretarias Executivas Regionais e abrigam atualmente 119 bairros em cinco distritos ${ }^{13}$.

A partir de 2008, as EEEPs foram instituídas no Ceará visando integrar o Ensino Médio à educação profissional em tempo integral. Para o estudo, a seleção foi feita por conglomerado em dois estágios. No primeiro estágio, foram selecionadas seis escolas por sorteio, uma em cada regional, com exceção da regional Centro que não possui. No segundo, foi selecionada uma turma por série (primeira a terceira), manhã ou tarde, totalizando três turmas por EEEPs. Foram recrutados, no mínimo, 13 adolescentes por turma.

Foram selecionados 291 adolescentes, entre 15 e 19 anos, frequentando da primeira a terceira série do Ensino Médio, matriculados no ano letivo de 2019 nas EEEPs selecionadas e que possuíssem smartphone, compondo uma amostra probabilística. Deste total, cinco foram retirados devido à ausência do preenchimento de um dos instrumentos de coleta ou dos dados antropométricos, sendo considerado perda amostral. Diante 
disso, a amostra final contou com o quantitativo de 286 adolescentes, distribuídos equitativamente entre as séries: 96 da primeira série, 95 da segunda série e 95 da terceira série do Ensino Médio.

Foram excluídos aqueles que não compareceram às aulas nos dias da coleta dos dados, com diagnóstico autorreferido de escoliose, fratura ou lesões degenerativas na coluna cervical, lesões traumáticas recentes e portadores de deficiência física por serem fatores relacionados à presença de alterações posturais e álgicas na coluna. Além disso, foram excluídas gestantes pelas mudanças fisiológicas, e portadores de deficiência visual, auditiva e cognitiva devido à falta de adaptabilidade dos instrumentos de coleta para essa população e ao autopreenchimento dos mesmos.

O quantitativo foi estimado por cálculo amostral, considerando uma população finita $(n=224.153)$ de adolescentes (15 a 19 anos) da cidade de Fortaleza ${ }^{12}$, prevalência de $18 \%$ de dor cervical em usuários de dispositivos móveis ${ }^{2}$, precisão amostral de 5\%, intervalo de confiança de 95\% e acréscimo de $10 \%$ para perda amostral. As séries e faixa etária elencadas para este estudo foram baseadas nas recomendações da Pesquisa Nacional de Saúde do Escolar (PENSE) ${ }^{14}$. A escola exerce importante influência na formação do adolescente que está em fase de desenvolvimento tanto cognitivo, social quanto emocional; Portanto, é um ambiente favorável para o monitoramento de fatores de riscos e proteção da população ${ }^{14}$.

Após as autorizações da direção das escolas, o recrutamento dos participantes iniciou-se por chamamento público para uma palestra explicativa dirigida aos pais/responsáveis e adolescentes. Em seguida, foi solicitado o consentimento através das assinaturas dos termos de consentimento livre e esclarecido (TCLE) e de assentimento.

A coleta de dados ocorreu em duas etapas. $\mathrm{Na}$ primeira etapa, eles responderam cinco instrumentos de coleta de dados autoaplicáveis, na própria sala de aula da escola, em horário determinado pela direção a fim de garantir a não interferência nas atividades escolares.

Os instrumentos de coleta aplicados foram: 1) Questionário sociodemográfico e condições de saúde; 2) Questionário Internacional de Atividade Física versão curta (International Physical Activity Questionnaire - IPAQ); 3) Questionário Nórdico de Sintomas Musculoesqueléticos (NMQ); 4) Self-Report Questionnaire (SRQ-20) e 5) Smartphone Addiction Inventory (SPAI-BR).

O questionário sociodemográfico e condições de saúde foi elaborado pelos pesquisadores, e coletou-se dados sociodemográficos (idade, sexo, raça, com quem reside, classe social, escolaridade materna e paterna) e histórico de saúde (características do sono e autoavaliação de saúde).

O IPAQ versão curta, validado no Brasil para aplicação em adultos, idosos e em adolescentes, é composto por oito questões abertas que estimam o tempo despendido por semana em diferentes dimensões de atividade física (caminhadas e esforços físicos de intensidades moderada e vigorosa) e de comportamento sedentário (posição sentada $)^{15}$. Para classificar o nível de atividade física foi utilizado neste estudo duas categorias: não sedentário (muito ativo, ativo, irregularmente ativo) e sedentário ${ }^{16}$.

O Questionário Nórdico de Sintomas Musculoesqueléticos, validado ${ }^{17}$ e adaptado transculturalmente $^{18}$ para português, mensura o relato de dor/sintomas musculoesqueléticos (sim/não) em nove regiões anatômicas (cervical, ombros, região torácica, cotovelos, punhos/mãos, região lombar, quadris/coxas, joelhos e tornozelos/pés) nos últimos sete dias.

O SRQ-20, validado para o português, é um instrumento que identifica sintomas psicossomáticos para o rastreamento de Transtorno Mental Comum (TMC) e reconhecido pela Organização Mundial da Saúde e validado no Brasil ${ }^{19}$. Possui vinte questões em quatro grupos de sintomas referidos nos últimos trinta dias com respostas $\operatorname{sim} /$ não. Neste estudo foram utilizados o somatório das respostas assinaladas como "sim" (variável numérica) e um ponto de corte de $\geq 8$ pontos para suspeita de TMC (variável categórica), com sensibilidade de $86,3 \%$ e especificidade de $89,3 \%{ }^{20}$.

O SPAI-BR é um instrumento com 26 itens, divididos em quatro subescalas, com resposta sim/não para avaliar a dependência do smartphone. O ponto de corte adotado para a dependência do smartphone foi de sete pontos, que possui sensibilidade de $90,54 \%$ e especificidade de 59,93\% para a validação e adaptação para o português ${ }^{21}$. Foi acrescentado ao final do instrumento duas perguntas sobre o tempo de uso do smartphone em horas na semana e no final de semana.

$\mathrm{Na}$ segunda etapa foi realizada a avaliação antropométrica (peso e altura) para cálculo do índice de massa corporal - IMC $\left(\mathrm{Kg} / \mathrm{cm}^{2}\right)$. O peso foi aferido mediante a utilização de balança digital portátil Omron, com capacidade de até 150 $\mathrm{Kg}$, calibrada e posicionada em superfície firme. Para mensuração da altura foi utilizado estadiômetro compacto portátil da marca Macrosul devidamente calibrado. O IMC foi classificado em presença ou ausência de excesso de peso, de 
acordo com tabela proposta pela Organização Mundial de Saúde ${ }^{22}$.

Os dados foram analisados pela estatística descritiva e inferencial usando o SPSS Statistics $\mathrm{IBM}^{\circledR}$ versão 23.0. $\mathrm{Na}$ análise univariada, foram calculados os valores absolutos (n) e relativos (\%) das variáveis categóricas, e as médias \pm desvio padrão (DP) das variáveis numéricas. $\mathrm{Na}$ análise dos fatores relacionados à dependência do smartphone aplicaram-se o Qui-quadrado de Pearson, seguido de cálculo do odds ratio bruto e respectivos intervalos de confiança de 95\%; e o teste t de Student, após teste de normalidade. Após, realizou-se a regressão logística multivariada, pelo método stepwise backward, selecionando as variáveis que apresentaram na análise bivariada o nível de significância de até $20 \%(\mathrm{p}<0,020)$, permitindo selecionar variáveis relevantes para o modelo e controlar variáveis de confusão, conforme proposto por Hosmer e Lemeshow ${ }^{23}$. No modelo final permaneceram as variáveis que apresentaram nível de significância de 5\% $(\mathrm{p}<0,05)$, com cálculo dos odds ratio ajustado e seus intervalos de confiança de $95 \%$.

O estudo foi aprovado pelo Comitê de Ética em Pesquisas em Seres Humanos da Universidade de Fortaleza - COÉTICA/UNIFOR, em consonância com a Resolução 466/12 do Conselho Nacional de Saúde. Pais/responsáveis e adolescentes consentiram sua participação pela assinatura dos termos de consentimento e assentimento.

\section{Resultados}

Sobre as variáveis socioeconômicas, houve maior proporção de adolescentes com 16 anos $(\mathrm{n}=90$; $31,5 \%)$, do sexo masculino ( $\mathrm{n}=152 ; 53,1 \%)$, da classe social D $(n=150 ; 52,4 \%)$ e genitores com Ensino Médio ( $\mathrm{n}=142 ; 49,6 \%$ para a mãe e $\mathrm{n}=127 ; 44,4 \%$ para o pai). Nas condições de saúde, dormiam em média 6,8 $( \pm 1,1)$ horas por dia, $40,2 \%(n=115)$ eram sedentários e $47,6 \%$ $(\mathrm{n}=136)$ relataram queixa de dor cervical e $23,1 \%(\mathrm{n}=66)$ tinham excesso de peso. Do total, $70,3 \%(n=201)$ apresentavam dependência do smartphone e o usavam por 5,8 horas $( \pm 3,5)$ e por 8,8 horas $( \pm 5,0)$ na semana e no final de semana, respectivamente (Tabela 1 ).

$\mathrm{Na}$ análise bivariada, a dependência do smartphone estava relacionada à queixa de dor nas regiões cervical $(\mathrm{OR}=2,960 ; \mathrm{p}=0,000)$, na parte superior das costas $(\mathrm{OR}=1,950 ; \mathrm{p}=0,010)$ e no punho $(\mathrm{OR}=1,956 ; \mathrm{p}=0,029)$, idade $(16,1 \pm 0,9$; $\mathrm{p}=0,008)$, horas de sono $(6,6 \pm 1,1 ; \mathrm{p}=0,001)$, tempo de uso no final de semana $(9,4 \pm 5,3$; $\mathrm{p}=0,000)$ e suspeita de TMC $(8,7 \pm 4,5 ; \mathrm{p}=0,000)$ (Tabela 2 e 3).

$\mathrm{Na}$ análise multivariada, a dependência do smartphone apresentou associação com a queixa de dor cervical ( $O R=2,206 ; p=0,020)$, menor idade $(\mathrm{OR}=0,583 ; \mathrm{p}=0,001)$, menos horas de sono $(\mathrm{OR}=0,715 ; \mathrm{p}=0,020)$, mais tempo de uso no final de semana $(O R=1,115 ; \mathrm{p}=0,015)$ e suspeita de TMC $(\mathrm{OR}=1,272 ; \mathrm{p}=0,000)$ (Tabela 4$)$.

\section{Discussão}

O presente estudo investigou um tema emergente, voltado para a área epidemiológica e de promoção da saúde, que envolve a dependência do smartphone pelos adolescentes e os fatores associados. Esta população em particular é fortemente atraída pela tecnologia, permanecendo por longas horas conectados à internet e redes sociais, entre outros ambientes virtuais ${ }^{11}$. Dentre os dispositivos móveis atuais, o smartphone é um dos principais meios de acesso à internet ${ }^{5}$.

Em virtude disso, há crescente preocupação na compreensão do comportamento e do vício da internet, videogames e smartphones. $\mathrm{O}$ uso da internet e a interação com conteúdos/atividades online são historicamente considerados como um vício global. Diante deste cenário, o surgimento do smartphone, com possibilidade de conexão e oferta de aplicativos, torna-se um dispositivo potencialmente viciante ${ }^{9}$.

Preocupada com essas questões, a OMS declara que o uso excessivo e problemático dos dispositivos eletrônicos, dentre eles o smartphone, constitui um problema de saúde pública mundial, e incentiva pesquisas para fornecer evidências científicas sobre os fatores implicados a este comportamento e os possíveis malefícios na saúde dos usuários ${ }^{11}$.

O uso problemático do smartphone pode ser pesquisado por instrumentos específicos que irão analisar a dependência/vício e pela mensuração do tempo de uso deste dispositivo. Neste estudo, ambas variáveis foram investigadas, constatando-se que 70,3\% da amostra apresentava dependência do smartphone e os tempos de uso eram de 5 horas e 48 minutos na semana e 8 horas e 48 minutos no final de semana. Além disso, os adolescentes com dependência do smartphone tinham mais tempo de uso no final de semana em relação àqueles que não dependentes.

A prevalência encontrada foi superior a outras reportadas com adolescentes em diferentes 
Tabela 1. Distribuição das variáveis investigadas dos adolescentes de escolas de tempo integral. Fortaleza, Ceará, 2019.

\begin{tabular}{|c|c|c|c|}
\hline Variáveis & $\mathbf{n}$ & $\%$ & $\begin{array}{c}\text { Média }+ \\
\text { DP }\end{array}$ \\
\hline \multicolumn{4}{|c|}{ Variáveis sociodemográficas } \\
\hline \multicolumn{4}{|l|}{ Idade } \\
\hline 15 & 80 & 28,0 & - \\
\hline 16 & 90 & 31,5 & - \\
\hline 17 & 88 & 30,8 & - \\
\hline 18 & 22 & 7,7 & - \\
\hline 19 & 6 & 2,1 & - \\
\hline \multicolumn{4}{|l|}{ Sexo } \\
\hline Masculino & 152 & 53,1 & - \\
\hline Feminino & 134 & 46,9 & - \\
\hline \multicolumn{4}{|l|}{ Raça autorreferida } \\
\hline Parda & 154 & 53,8 & - \\
\hline Branca & 72 & 25,2 & - \\
\hline Preta & 39 & 13,6 & - \\
\hline Amarela & 12 & 4,2 & - \\
\hline Indígena & 9 & 3,1 & - \\
\hline \multicolumn{4}{|l|}{ Reside com } \\
\hline Pai e mãe & 178 & 62,2 & - \\
\hline Somente com a mãe & 77 & 26,9 & - \\
\hline Somente com o pai & 4 & 1,4 & - \\
\hline Outros & 27 & 9,4 & - \\
\hline \multicolumn{4}{|l|}{ Classe social } \\
\hline A & 3 & 1,0 & - \\
\hline B & 6 & 2,1 & - \\
\hline $\mathrm{C}$ & 37 & 12,9 & - \\
\hline $\mathrm{D}$ & 150 & 52,4 & - \\
\hline E & 90 & 31,5 & - \\
\hline \multicolumn{4}{|l|}{ Escolaridade materna } \\
\hline Ensino Fundamental & 80 & 28 & - \\
\hline Ensino Médio & 142 & 49,6 & - \\
\hline Ensino Superior & 50 & 17,5 & - \\
\hline Não sabe informar & 14 & 4,9 & - \\
\hline \multicolumn{4}{|l|}{ Escolaridade paterna } \\
\hline Ensino Fundamental & 95 & 33,2 & - \\
\hline Ensino Médio & 127 & 44,4 & - \\
\hline Ensino Superior & 25 & 8,8 & - \\
\hline Não sabe informar & 39 & 13,6 & - \\
\hline \multicolumn{4}{|l|}{ Condições de saúde } \\
\hline \multicolumn{4}{|l|}{ Dorme bem } \\
\hline Não & 195 & 68,2 & - \\
\hline Sim & 91 & 31,8 & - \\
\hline Horas de sono & & & $6,8 \pm 1,1$ \\
\hline
\end{tabular}

Tabela 1. Distribuição das variáveis investigadas dos adolescentes de escolas de tempo integral. Fortaleza, Ceará, 2019.

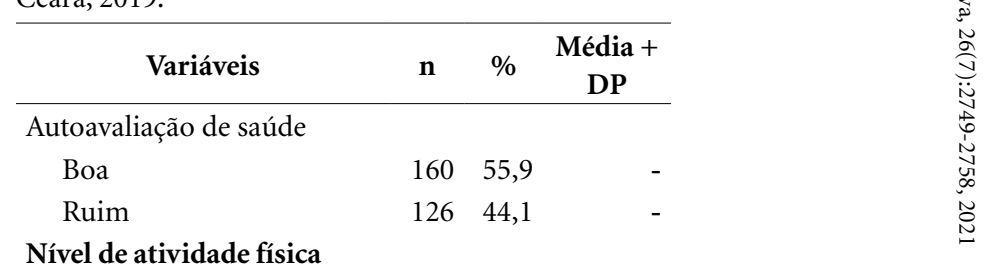

$$
\text { Q }
$$

ivel de atividade física

Não sedentário

Sedentário

$171 \quad 59,8$

Queixa de dor

Cervical

Não

Sim

$115 \quad 40,2$

Ombros

Não

Sim

Superior das costas

não

Sim

Inferior das costas

Não

Sim

Cotovelo

Não

Sim

Punho

Não

Sim

$150 \quad 52,4$

13647,6

$196 \quad 68,5$

$90 \quad 31,5$

$135 \quad 47,2$

15152,8

$141 \quad 49,3$

$145 \quad 50,7$

27696,5

$10 \quad 3,5$

20371,0

8329,0

Suspeita de Transtorno

Mental Comum

$\begin{array}{llll}\text { Não } & 136 & 47,6 & \text { - } \\ \text { Sim } & 150 & 52,4 & \text { - } \\ \text { Uso do smartphone } & & & \end{array}$

Uso do smartphone

Dependência do smartphone

$\begin{array}{lrl}\text { Não } & 85 & 29, \\ \text { Sim } & 201 & 70,\end{array}$

Tempo de uso do

smartphone na semana

Tempo de uso do

smartphone no final de

semana

\section{Excesso de peso}

\begin{tabular}{lrrr} 
Não & 220 & 79,9 & - \\
Sim & 66 & 23,1 & - \\
\hline DP: desvio padrão. & & & \\
Fonte: Dados do estudo, 2019.
\end{tabular}

países, de 55\% em Taiwan ${ }^{24}, 50,6 \%$ na Turquia ${ }^{25}$, $30,9 \%$ na Coréia do Sul ${ }^{6}$ e $16,9 \%$ na Suíça ${ }^{26}$. No Brasil, estudo prévio para adaptação e validação do Smartphone Addiction Inventory aponta uma prevalência de 35\% em uma população jovem (18-25 anos $)^{21}$. Acredita-se que este valor eleva- 
Tabela 2. Análise bivariada da associação entre a dependência do smartphone e as variáveis sexo, condições de saúde, nível de atividade, queixa de dor e excesso de peso dos adolescentes de escolas de tempo integral. Fortaleza, Ceará, 2019.

\begin{tabular}{|c|c|c|c|c|}
\hline \multirow{3}{*}{ Variáveis } & \multirow{2}{*}{\multicolumn{2}{|c|}{$\begin{array}{c}\text { Dependência do smartphone } \\
\text { n (\%) }\end{array}$}} & \multirow{3}{*}{ OR bruto (IC95\%) } & \multirow{3}{*}{ p-valor } \\
\hline & & & & \\
\hline & não & $\operatorname{sim}$ & & \\
\hline Sexo & & & & $0,131^{\mathrm{a}}$ \\
\hline Masculino & $51(60,0)$ & $101(50,2)$ & 1 & \\
\hline Feminino & $34(40,0)$ & $100(49,8)$ & $1,485(0,888-2,484)$ & \\
\hline Dorme bem & & & & $0,050^{\mathrm{a}}$ \\
\hline Sim & $20(23,5)$ & $71(35,3)$ & 1 & \\
\hline Não & $65(76,5)$ & $130(64,7)$ & $1,775(0,995-3,166)$ & \\
\hline Autoavaliação de saúde & & & & $0,156^{\mathrm{a}}$ \\
\hline Boa & $53(62,4)$ & $107(53,2)$ & 1 & \\
\hline Ruim & $32(37,6)$ & $94(46,8)$ & $1,455(0,866-2,445)$ & \\
\hline Nível de atividade física & & & & $0,058^{\mathrm{a}}$ \\
\hline Não sedentário & $58(68,2)$ & $113(56,2)$ & 1 & \\
\hline Sedentário & $27(31,8)$ & $88(43,8)$ & $1,673(0,980-2,856)$ & \\
\hline \multicolumn{5}{|l|}{ Queixa de dor } \\
\hline Cervical & & & & $0,000^{*}$ \\
\hline Não & $60(70,6)$ & $90(44,8)$ & 1 & \\
\hline Sim & $25(29,4)$ & $111(55,2)$ & $2,960(1,720-5,095)$ & \\
\hline Ombro & & & & $0,109^{a}$ \\
\hline Não & $64(75,3)$ & $132(65,7)$ & 1 & \\
\hline Sim & $21(24,7)$ & $69(34,3)$ & $1,593(0,899-2,824)$ & \\
\hline Superior das costas & & & & $0,010^{*}$ \\
\hline Não & $50(58,8)$ & $85(42,3)$ & 1 & \\
\hline Sim & $35(41,2)$ & $116(57,7)$ & $1,950(1,165-3,262)$ & \\
\hline Inferior das costas & & & & $0,066^{\mathrm{a}}$ \\
\hline Não & $49(57,6)$ & $92(45,8)$ & 1 & \\
\hline Sim & $36(42,4)$ & $109(54,2)$ & $1,613(0,966-2,691)$ & \\
\hline Cotovelo & & & & $0,153^{\mathrm{a}}$ \\
\hline Não & $80(94,1)$ & $196(97,5)$ & 1 & \\
\hline Sim & $5(5,9)$ & $5(2,5)$ & $0,408(0,115-1,448)$ & \\
\hline Punho & & & & $0,029^{*}$ \\
\hline Não & $68(80,0)$ & $135(67,2)$ & 1 & \\
\hline Sim & $17(20,0)$ & $66(32,8)$ & $1,956(1,065-3,590)$ & \\
\hline Excesso de peso & & & & $0,098^{\mathrm{a}}$ \\
\hline Sem excesso de peso & $60(70,6)$ & $160(79,6)$ & 1 & \\
\hline Com excesso de peso & $25(29,4)$ & $41(20,4)$ & $0,615(0,345-1,097)$ & \\
\hline
\end{tabular}

do possa ser justificado pelo aumento do acesso à internet e aos seus recursos pelos adolescentes brasileiros, destacadamente pelos seus smartphones ${ }^{5,14}$. Ademais, ao utilizar um instrumento adaptado transculturalmente para a língua portuguesa se mitiga os riscos de vieses.

O tempo de uso do smartphone pelos adolescentes deve ser também monitorado devido a sua relação direta com a dependência deste dispositivo $^{27}$. Estudos prévios na Coréia ${ }^{6}$ e na Suíça ${ }^{26}$ evidenciaram tempos de uso similares aos encontrados na presente pesquisa, com médias de 5,2 horas e 5,6 horas, respectivamente. Diferente dos dados anteriores, tempo de uso inferior foi encontrado em estudo realizado no Reino Unido com tempo gasto de 3,1 horas/dia ${ }^{28}$. 
Tabela 3. Análise bivariada da relação entre a dependência do smartphone e as variáveis idade, horas de sono, uso do smartphone e suspeita de transtorno mental comum dos adolescentes de escolas de tempo integral. Fortaleza, Ceará, 2019.

\begin{tabular}{lccc}
\hline \multirow{2}{*}{ Variáveis } & \multicolumn{2}{c}{$\begin{array}{c}\text { Dependência do smartphone } \\
\text { (média } \pm \text { DP) }\end{array}$} & \multirow{2}{*}{ p-valor } \\
\cline { 2 - 3 } & \multicolumn{1}{c}{ Não } & Sim & \\
\hline Idade (anos) & $16,5 \pm 1,1$ & $16,1 \pm 0,9$ & $0,008^{\star}$ \\
Horas de sono & $7,1 \pm 1,1$ & $6,6 \pm 1,1$ & $0,001^{\star}$ \\
Tempo de uso do smartphone na semana & $5,7 \pm 3,5$ & $5,9 \pm 3,5$ & 0,613 \\
Tempo de uso do smartphone no fim de semana & $7,3 \pm 3,6$ & $9,4 \pm 5,3$ & $0,000^{\star}$ \\
Transtorno Mental Comum & $4,9 \pm 3,4$ & $8,7 \pm 4,5$ & $0,000^{*}$ \\
\hline
\end{tabular}

DP: desvio padrão.

Fonte: Dados do estudo, 2019.

Tabela 4. Análise multivariada da relação entre a dependência do smartphone com as variáveis do estudo em adolescentes de escolas de tempo integral. Fortaleza, Ceará, 2019.

\begin{tabular}{|c|c|c|}
\hline Variáveis & $\begin{array}{l}\text { OR ajustado } \\
\text { (IC95\%) }\end{array}$ & $\begin{array}{c}\text { p- } \\
\text { valor }\end{array}$ \\
\hline Sexo (feminino) & $0,600(0,305-1,183)$ & 0,140 \\
\hline Dorme bem (não) & $0,573(0,258-1,270)$ & 0,170 \\
\hline $\begin{array}{l}\text { Autoavaliação de } \\
\text { saúde (ruim) }\end{array}$ & $0,685(0,349-1,348)$ & 0,274 \\
\hline $\begin{array}{l}\text { Prática de atividade } \\
\text { física (sedentário) }\end{array}$ & $1,662(0,855-3,232)$ & 0,134 \\
\hline \multicolumn{3}{|l|}{ Queixa de dor } \\
\hline Cervical (sim) & $2,206(1,131-4,302)$ & $0,020^{*}$ \\
\hline Ombro (sim) & $1,085(0,507-2,324)$ & 0,833 \\
\hline $\begin{array}{l}\text { Superior das costas } \\
(\operatorname{sim})\end{array}$ & $1,533(0,770-3,052)$ & 0,223 \\
\hline $\begin{array}{l}\text { Inferior das costas } \\
(\operatorname{sim})\end{array}$ & $1,025(0,537-1$ & 0,939 \\
\hline Cotovelo (sim) & $0,526(0,110-2,506)$ & 0,420 \\
\hline Punho (sim) & $1,137(0,540-2,395)$ & 0,735 \\
\hline Idade & $0,583(0,426-0,799)$ & $0,001^{\star}$ \\
\hline Horas de sono & $0,715(0,538-0,949)$ & $0,020^{\star}$ \\
\hline $\begin{array}{l}\text { Tempo de uso do } \\
\text { smartphone no final } \\
\text { de semana }\end{array}$ & $1,115(1,022-1,217)$ & $0,015^{\star}$ \\
\hline $\begin{array}{l}\text { Transtorno mental } \\
\text { comum }\end{array}$ & $1,272(1,150-1,407)$ & $0,000^{*}$ \\
\hline Excesso de peso (sim) & $0,905(0,411-1,994)$ & 0,804 \\
\hline
\end{tabular}

OR: odds ratio; IC95\%: intervalo de confiança de 95\%. Parâmetros do modelo final: Cox \& Snell $=0,259$; Nagelkerke $=$ 0,$368 ; \chi^{2}$ do modelo $=85,854 .{ }^{*} \mathrm{p}<0,05$.

Fonte: Dados do estudo, 2019.

No presente estudo, verificou-se que a dependência do smartphone estava associada à idade.
Em consonância com a idade, pesquisa na Suíça constatou que o vício em smartphones foi mais prevalente em adolescentes mais jovens ( 15 a 16 anos) em comparação com adultos jovens (19 anos ou mais $)^{26}$. Sabe-se que esta geração enfrenta diferentes estressores mais do que qualquer outra geração anterior. Além das mudanças fisiológicas inerentes à adolescência, há uma cobrança da exposição do seu cotidiano no ambiente virtual, exigindo um maior tempo online. $\mathrm{O}$ uso excessivo do smartphone e dos jogos online são tentativas de minimizar estressores, como as demandas escolares, as incertezas do futuro, as pressões familiares e sociais, levando essa população a comportamentos viciantes ${ }^{8}$.

Outros fatores também estavam associados à dependência do smartphone como menos horas de sono, dor cervical e transtorno mental comum. Sobre os aspectos da saúde física e mental, estudo de revisão elencou os múltiplos problemas de saúde decorrentes do uso excessivo do smartphone, como depressão, ansiedade, impulsividade, comportamento sedentário, distúrbio do sono, contratura na musculatura e dor na coluna cervical e lombar8.

Várias pesquisas abordam a associação da dependência do smartphone com a qualidade do sono. Dentre eles, estudos na Suíça ${ }^{29}$ e no Brasil ${ }^{30}$ apontaram que os usuários de smartphones dormem menos, especialmente nos dias úteis, como também relatam mais problemas de sono. Outro estudo, na Indonésia, revelou que o uso excessivo do smartphone à noite pode causar distúrbios no sono e contribui para o surgimento de depressão ${ }^{27}$. Os impactos do uso excessivo deste dispositivo na qualidade do sono e na sua duração são preocupantes, pois a restrição do sono pode causar transtornos físicos e mentais, o que torna importante o uso consciente e monitorado. 
Dentre as repercussões físicas relacionadas ao desfecho, os adolescentes da amostra reportaram à presença de dor na região cervical. Queixa similar foi relatada pelos adolescentes de Taiwan que informaram desconforto musculoesquelético na coluna cervical e torácica quando utilizavam o smartphone por mais de três horas diárias ${ }^{31}$. Outra pesquisa, no Líbano, encontrou relação entre a presença da dor musculoesquelética na cervical e na região das costas com o uso dos smartphones e/ou tablets em virtude da flexão excessiva, após cinco a sete horas nesses dispositivos ${ }^{32}$. Como também na China, em adolescentes com idade média de 18 anos, em que a região do corpo que apresentava maior queixa álgica foi a cervical após o uso prolongado do smartphone ${ }^{33}$.

Em relação ao aspecto da saúde mental, detectou-se que $52,4 \%$ da amostra tinha suspeita de TMC e que havia relação com o desfecho de dependência do smartphone. Pesquisas anteriores realizadas na Hungria ${ }^{34}$ e Turquia ${ }^{25}$ concluíram que o tempo de uso do smartphone tem relação direta com a impulsividade, a ansiedade, a depressão, a sensibilidade interpessoal e com os sintomas de hostilidade. De forma convergente, outros dois estudos transversais realizados no Japão ${ }^{35}$ e na Coreia ${ }^{36}$ atestam que, quanto maior tempo de uso, maior é o risco de ocorrência de sintomas depressivos e pensamentos suicidas.

Diante dos achados, alerta-se para a importância do monitoramento do uso excessivo do smartphone pelos adolescentes e dos possíveis malefícios à saúde mental e física; e, ainda, destaca-se a necessidade de implantação de programas de prevenção/cuidados à saúde direcionados para essas questões com envolvimento da família, dos ambientes educacionais e do sistema de saúde. A Sociedade Brasileira de Pediatra reforça a conscientização das leis vigentes no país e a implementação de políticas públicas, que culminem em campanhas de educação em saúde e materiais de apoio, destinadas à proteção integral e à prevenção dos riscos do uso de internet, redes sociais, jogos e outros aplicativos ${ }^{37}$.

Reconhecem-se algumas limitações neste estudo no quesito amostra, como a não inclusão de escolas da rede privada dificultando análises que envolvessem fatores socioeconômicos, e a delimitação da faixa etária (15-19 anos). Apesar dessas limitações potencialmente afetarem generalizações dos resultados, acredita-se que os achados da pesquisa contribuirão para a discussão do tema.

\section{Conclusão}

Neste estudo, que buscou avaliar a dependência do smartphone e os fatores associados em adolescentes, foi constatada elevada prevalência nos adolescentes da amostra e sua relação com múltiplos fatores, como idade, tempo de uso e comprometimento das condições de saúde física, evidenciado pela queixa de dor cervical, além de prejuízos à saúde mental com a redução de horas de sono e presença de transtorno mental comum.

Os resultados encontrados podem ser elucidados pelas particularidades inerentes à adolescência como a rotina de estudos e necessidade do uso de internet para fins de pesquisa, bem como a sua utilização para momentos de lazer como assistir filme, jogos e relacionar-se com outras pessoas. Desse modo, a população estudada pode desenvolver este distúrbio comportamental em virtude destas particularidades.

Portanto, diante dos resultados destaca-se a importância de campanhas educativas e de promoção da saúde em diversos cenários socioeducativos e na mídia, com intuito de sensibilizar os adolescentes, pais, educadores e profissionais de saúde quanto os riscos da dependência do smartphones à saúde física e mental e conscientizar quanto a utilização adequada deste dispositivo. 


\section{Colaboradores}

PPB Nunes, APV Abdon, CB Brito, FVM Silva e DQ Martins responderam pela contribuição substancial na concepção e planejamento, na aquisição, análise e interpretação de dados; na redação e elaboração do artigo; e na aprovação da versão final. ICA Santos e PMF Meira, pela contribuição substancial na aquisição de dados, na redação e elaboração do artigo; e na aprovação da versão final. MA Frota, na contribuição substancial na concepção e planejamento; na redação e elaboração do artigo; e na aprovação da versão final.

\section{Agradecimentos}

Ao Conselho Nacional de Desenvolvimento Científico e Tecnológico (CNPq) e à Universidade de Fortaleza (Unifor) pela Fundação Edson Queiroz pelas bolsas de iniciação científicas.

À Secretaria de Educação do Estado do Ceará (SEDUC) e às Escolas Estaduais de Educação Profissional (EEEPs) de Ensino Médio da cidade de Fortaleza pelo apoio.

\section{Referências}

1. Spizzirri RCP, Wagner A, Mosmann CP, Armani AB. Adolescência conectada: mapeando o uso da internet em. Psicol Argumento 2012; 30(69):327-335.

2. Xie Y, Szeto G, Dai J. Prevalence and risk factors associated with musculoskeletal complaints among users of mobile handheld devices: a systematic review. Appl Ergon 2017;59:132-142.

3. Pew Research Center. Smartphone ownership and internet usage continues to climb in emerging economies. Pew Research Center [Internet]. 2016 Feb 22. [cited 2020 Apr 26];1-5. Available from: http://www. pewglobal.org/2016/02/22/smartphone-ownership -and-internet-usage-continues-to-climb-in-emerging-economies/

4. We are social. Global Digital Report 2019 - We Are Social [Internet]. 2019 [cited 2020 Apr 26]. p. 1-11. Available from: https://wearesocial.com/global-digital-report-2019

5. Brazilian Internet Steering Committee. Pesquisa sobre o uso da internet por crianças e adolescentes no brasil. [Internet]. Núcleo de Informação e Coordenação do Ponto BR, editor. Pesquisa sobre o uso da internet por crianças e adolescentes no Brasil: TIC kids online Brasil 2018 / Survey on internet use by children in Brazil: ICT kids online Brazil 2018. São Paulo; 2019.352 p.

6. Cha SS, Seo BK. Smartphone use and smartphone addiction in middle school students in Korea: Prevalence, social networking service, and game use. Heal Psychol Open 2018; 5(1):1-25.

7. Elhai JD, Dvorak RD, Levine JC, Hall BJ. Problematic smartphone use: A conceptual overview and systematic review of relations with anxiety and depression psychopathology. J Affect Disord 2017; 207:251-259.

8. Derevensky JL, Hayman V, Lynette Gilbeau. Behavioral Addictions: Excessive Gambling, Gaming, Internet, and Smartphone Use Among Children and Adolescents. Pediatr Clin North Am 2019; 66(6):1163-1182.

9. Gutiérrez JDS, de Fonseca FR, Rubio G. Cell-phone addiction: A review. Front Psychiatry 2016; 7(175):115.

10. Csibi S, Griffiths MD, Cook B, Demetrovics Z, Szabo A. The Psychometric Properties of the Smartphone Application-Based Addiction Scale (SABAS). Int J Ment Health Addict 2018; 16(2):393-403.

11. World Health Organization (WHO). Public health implications of excessive use of the internet, computers, smartphones and similar electronic devices: meeting report, Main Meeting Hall, Foundation for Promotion of Cancer Research, National Cancer Research Centre, Tokyo, Japan, 27-29 August 2014. [Internet]. 2015[cited 2021 Mar 9]. Available from: https://apps.who.int/iris/bitstream/handle/10665/184264/9789241509367_eng.pdf?sequen$\mathrm{ce}=1$ \&isAllowed $=\mathrm{y}$

12. Instituto Brasileiro de Geografia e Estatística (IBGE). Panorama população. Fortaleza, Ceará [Internet]. 2010 [acessado 2020 abr 26]. Disponível em: https:// cidades.ibge.gov.br/brasil/ce/fortaleza/panorama

13. Prefeitura de Fortaleza. A cidade [Internet]. 2014 [acessado 2020 abr 26]. p. 289. Disponível em: www. fortaleza.ce.gov.br/a-cidade

14. Instituto Brasileiro de Geografia e Estatística (IBGE). Pesquisa Nacional de Saúde do Escolar 2015. Brasília: IBGE; 2016. 132p. 
15. Pinto Guedes D, Correa Lopes C, Pinto Guedes JER. Reprodutibilidade e validade do questionário internacional de atividade física em adolescentes. Rev Bras Med do Esporte 2005;11(2):151-158.

16. Ceschini FL, Figueira Júnior A. Nível de atividade física de adolescentes durante o ensino médio. Rev Bras Cien Saude 2006; 3(7):32-38.

17. Pinheiro FA, Tróccoli BT, Carvalho CV. Validação do Questionário Nórdico de Sintomas Osteomusculares como medida de morbidade. Rev Saude Publica 2002; 36(3):307-312.

18. Barros ENC, Alexandre NMC. Cross-cultural adaptation of the Nordic musculoskeletal questionnaire. Int Nurs Rev 2003;50(2):101-108.

19. Mari JJ, Williams P. A Validity Study of a Psychiatric Screening Questionnaire. Br J Psychiatry 1986; 148:23-27.

20. Moraes RSM, Silva DAS, Oliveira WF, Peres MA. Iniquidades sociais na prevalência de desordens mentais comuns em adultos: Estudo de base populacional no Sul do Brasil. Rev Bras Epidemiol 2017; 20(1):43-56.

21. Khoury JM, Freitas AAC, Roque MAV, Albuquerque MR, Neves MDCL, Garcia FD. Assessment of the accuracy of a new tool for the screening of smartphone addiction. PLoS One 2017;12(5):1-13.

22. Brasil. Ministério de Saúde (MS). Saúde IMC [Internet]. 2017 [acessado 2020 abr 20]. Brasília: MS. Disponível em: https://www.saude.gov.br/artigos/ 804-imc/40510-imc-em-criancas-e-adolescentes

23. Bursac Z, Gauss CH, Williams DK, Hosmer DW. Purposeful selection of variables in logistic regression. Source Code Biol Med 2008; 3:1-8.

24. Liu CH, Lin SH, Pan YC, Lin YH. Smartphone gaming and frequent use pattern associated with smartphone addiction. Med 2016 1; 95(28):1-4.

25. Fırat S, Gül H, Sertçelik M, Gül A, Gürel Y, Kılıç BG. The relationship between problematic smartphone use and psychiatric symptoms among adolescents who applied to psychiatry clinics. Psychiatry Res 2018; 270:97-103.

26. Haug S, Paz Castro R, Kwon M, Filler A, Kowatsch T, Schaub MP. Smartphone use and smartphone addiction among young people in Switzerland. J Behav Addict 2015; 4(4):299-307.

27. Dewi RK, Efendi F, Has EMM, Gunawan J. Adolescents' smartphone use at night, sleep disturbance and depressive symptoms. Int J Adolesc Med Health 2018; 33(2):1-8.

28. Hussain Z, Griffiths MD, Sheffield D. An investigation into problematic smartphone use: The role of narcissism, anxiety, and personality factors. J Behav Addict 2017;6(3):378-386.
29. Schweizer A, Berchtold A, Barrense-Dias Y, Akre C, Suris JC. Adolescents with a smartphone sleep less than their peers. Eur J Pediatr 2017;176(1):131-136.

30. Caumo GH, Spritzer D, Carissimi A, Tonon AC. Exposure to electronic devices and sleep quality in adolescents: A matter of type, duration, and timing. Sleep Heal 2020; 2020; 6(2):172-178.

31. Yang SY, Chen M De, Huang YC, Lin CY, Chang $\mathrm{JH}$. Association between smartphone use and musculoskeletal discomfort in adolescent students. $J$ Community Health 2016; 42(3):423-430.

32. Fares J, Fares MY, Fares Y. Musculoskeletal neck pain in children and adolescents: Risk factors. Surg Neurol Int 2017; 8(72):1-5.

33. Namwongsa S, Puntumetakul R, Neubert MS, Boucaut R. Factors associated with neck disorders among university student smartphone users. Work 2018; 61(3):367-378.

34. Körmendi A. Smartphone usage among adolescents. Psychiatr Hung 2015; 30(3):297-302.

35. Nishida T, Tamura H, Sakakibara H. The association of smartphone use and depression in Japanese adolescents. Psychiatry Res 2019; 273:523-527.

36. Kim H, Cho M-K, Ko H, Yoo JE, Song Y-M. Association between Smartphone Usage and Mental Health in South Korean Adolescents: The 2017 Korea Youth Risk Behavior Web-Based Survey. Korean J Fam Med 2020; 41(2):98-104.

37. Sociedade Brasileira de Pediatria (SBP). Saúde de Crianças e Adolescentes na Era Digital. Manual de Orientação. No 1. 2016 Out.[cited 20 Apr 2020]. 1-13 p. Available from: https://www.sbp.com.br/fileadmin/ user_upload/2016/11/19166d-MOrient-Saude-Crian -e-Adolesc.pdf

Artigo apresentado em 29/04/2020

Aprovado em 19/04/2021

Versão final apresentada em 21/04/2021

Editores-chefes: Romeu Gomes, Antônio Augusto Moura da Silva 